\title{
Grand Challenges in Astrochemistry
}

\author{
Cristina Puzzarini ${ }^{1,2 *}$ \\ 'Dipartimento di Chimica "Giacomo Ciamician", University of Bologna, Bologna, Italy, ${ }^{2}$ Interuniversity Center "Systems and \\ Theories for Astrochemical Research-STAR", Scuola Normale Superiore, Pisa, Italy
}

Keywords: astrochemistry, ISM, interstellar molecules, COM, PAH, chemical evolution

Astrochemistry and molecular astrophysics are often used as synonyms to define an interdisciplinary field that involves chemistry and astronomy, (astro)physics, as well as a "flavor" of biology and geology. Even if it is difficult to define an unique goal, it can be surely affirmed that the main aim is to understand the chemical evolution occurring in space: from diatomics to molecules of a certain degree of complexity and beyond. In other words, this research area studies how molecules are formed and destroyed in different astronomical environments as well as how they interact with radiation. To summarize (and, at the same time, simplify), the focus of astrochemistry is the investigation of chemical processes taking place in space, including molecular evolution and complexity. Molecules have been (and still are being) found everywhere in space: in the interstellar medium, in circumstellar shells, in pregalactic gas, in protostellar disks, and in the atmospheres of planets and stars. Molecules are thus ubiquitous and they can be considered unique probes of molecular excitation mechanisms, radiative transfer, and kinematics.

As is well-known, most of the matter-energy content of the Universe is composed of dark energy and dark matter. Indeed, atoms and molecules contribute $<5 \%$ of the total. Focusing on atomic elements, the contribution of hydrogen and helium amounts to about $98 \%$; therefore, that of heavier elements (such as carbon, nitrogen, and oxygen) is only about $2 \%$. Nevertheless, this small fraction of heavy elements makes possible a great variety of chemical compounds. In the disk of our Galaxy (the Milky Way), about $90 \%$ of the atomic/molecular mass is in stars, and the remaining is in the interstellar matter, mainly in the form of clouds. These consist of gas and tiny dust particles and are the components out of which new stars and planets are born.

When do we place the birth of astrochemistry? At the time of the discovery of interstellar gas (Hartmann, 1904) and dust (Trumpler, 1930), it has thought that the extreme conditions (temperatures ranging between 10 and $10^{6} \mathrm{~K}$ and densities from $10^{-4}$ to $10^{8}$ particles $/ \mathrm{cm}^{3}$ ) of the interstellar medium (ISM) would only allow the presence of atoms. In the early forties, however, McKellar identified spectral lines attributable to diatomic molecules (McKellar, 1940), undermining the belief in the absence of molecular reactivity in the ISM. It was only in the late sixties, after the birth of radioastronomy, that the first polyatomic molecules were identified. The first molecules discovered were: ammonia $\left(\mathrm{NH}_{3}\right)$ in 1968 (Cheung et al., 1968); water $\left(\mathrm{H}_{2} \mathrm{O}\right)$ (Cheung et al., 1969) and formaldehyde $\left(\mathrm{H}_{2} \mathrm{CO}\right)$, the first organic molecule (Snyder et al., 1969), in 1969; hydrocyanic acid (HCN) (Snyder and Buhl, 1971), carbon monoxide (CO) (Wilson et al., 1970) and methanol $\left(\mathrm{CH}_{3} \mathrm{OH}\right)$ in 1970 (Wilson et al., 1970); formic acid (HCOOH) (Snyder and Buhl, 1971) and formamide $\left(\mathrm{H}_{2} \mathrm{NCHO}\right)$ (Snyder and Buhl, 1971) in 1971. Since then, more than 200 molecules have been detected in the ISM and circumstellar shells and the rate of discovery continues at rapid pace. Interestingly, the molecules detected by radioastronomy, which range in size from diatomics up to 13 atoms, are overwhelmingly organic in nature. Particularly fascinating are the so-called "complex organic molecules" (COMs) (Herbst and van Dishoeck, 2009), which are defined as molecules containing more than 5 atoms and including at least one carbon atom.

By the late 1960s and the early 1970s, it had become clear that the ISM is host to a rich chemistry, leading to the emergence of a new branch of science: Astrochemistry. Over the years, the discovery of new molecules has led to questions about how these molecules could be formed in such hostile environments and how far chemical complexity can go. In parallel, further questions arose: How 
are molecules incorporated into stars and planets? Is the chemistry occurring in the ISM related to the origin of life on Earth? All these questions and the related need to deepen our knowledge represent the current frontiers in astrochemistry.

The considerations above lead to the definition of two grand challenges. (1) The census of the interstellar molecules and beyond, where "beyond" means the search for molecules in (exo)planets and other astronomical objects (e.g., comets). (2) The mechanisms of formation of molecules and the chemical evolution of astronomical objects. Together with these two grand challenges, we can envisage a third one: (3) the origin of life. Indeed, understanding the chemical evolution can help to gain insight into the emergence of life on Earth. The list above is not exhaustive but, rather, a selection of major themes that intrinsically contain (or are related to) further challenges. In this respect, a comprehensive overview is presented in van Dishoeck (2017).

\section{GRAND CHALLENGE 1: ON THE HUNT FOR MOLECULES}

The progress of observational studies revealed chemical diversity in space, the source-to-source variation in chemical composition which led to the concept of chemical evolution. In order to derive the chemical composition of an astronomical object a synergic interplay of radioastronomical observations and laboratory spectroscopy is required (see e.g., Müller et al., 2005; Belloche et al., 2014; McGuire et al., 2016; McGuire, 2018; Melosso et al., 2018). Indeed, the unequivocal proof of the presence of a given molecule in the astronomical environment under consideration is provided by the astronomical observation of its spectroscopic features (Tennyson, 2005; Yamamoto, 2017), with the overwhelming majority of gas-phase chemical species being identified via their rotational signatures (McGuire, 2018). The intensity of the observed/assigned lines needs then to be converted into a species abundance (Arthurs et al., 1960; Nash, 1990; Goldsmith and Langer, 1999), whose interpretation requires comparison with model predictions (e.g., Garrod et al., 2008; Holdship et al., 2017). These processes and their interconnection are sketched in Figure 1, and require a strong interplay of different communities: astronomy, astrophysics, and chemistry; experimentalists and theoreticians.

Despite the more than 200 molecules that have been discovered in the ISM and circumstellar shells, a significant number of features in radioastronomical spectra are still unidentified. This means that we are far from a complete census of the interstellar molecules. How many molecules have escaped our knowledge? And what about chemical complexity? Among the molecules discovered in space, those of prebiotic character are of particular relevance. In the last two decades, a great effort has been put in order to demonstrate the presence of biological building-block molecules in the ISM, because they can provide important information on the Galactic chemical evolution. To give an example, glycine $\left(\mathrm{NH}_{2} \mathrm{CH}_{2} \mathrm{COOH}\right)$, the simplest amino acid, has been intensively searched for in the ISM (see Kuan et al., 2003 and references therein). While it has been found (together with many other amino acids and nucleic bases) in some meteorites fallen on Earth (e.g., Murchison meteorite; Kvenvolden et al., 1970) and in the coma of comets (e.g., the

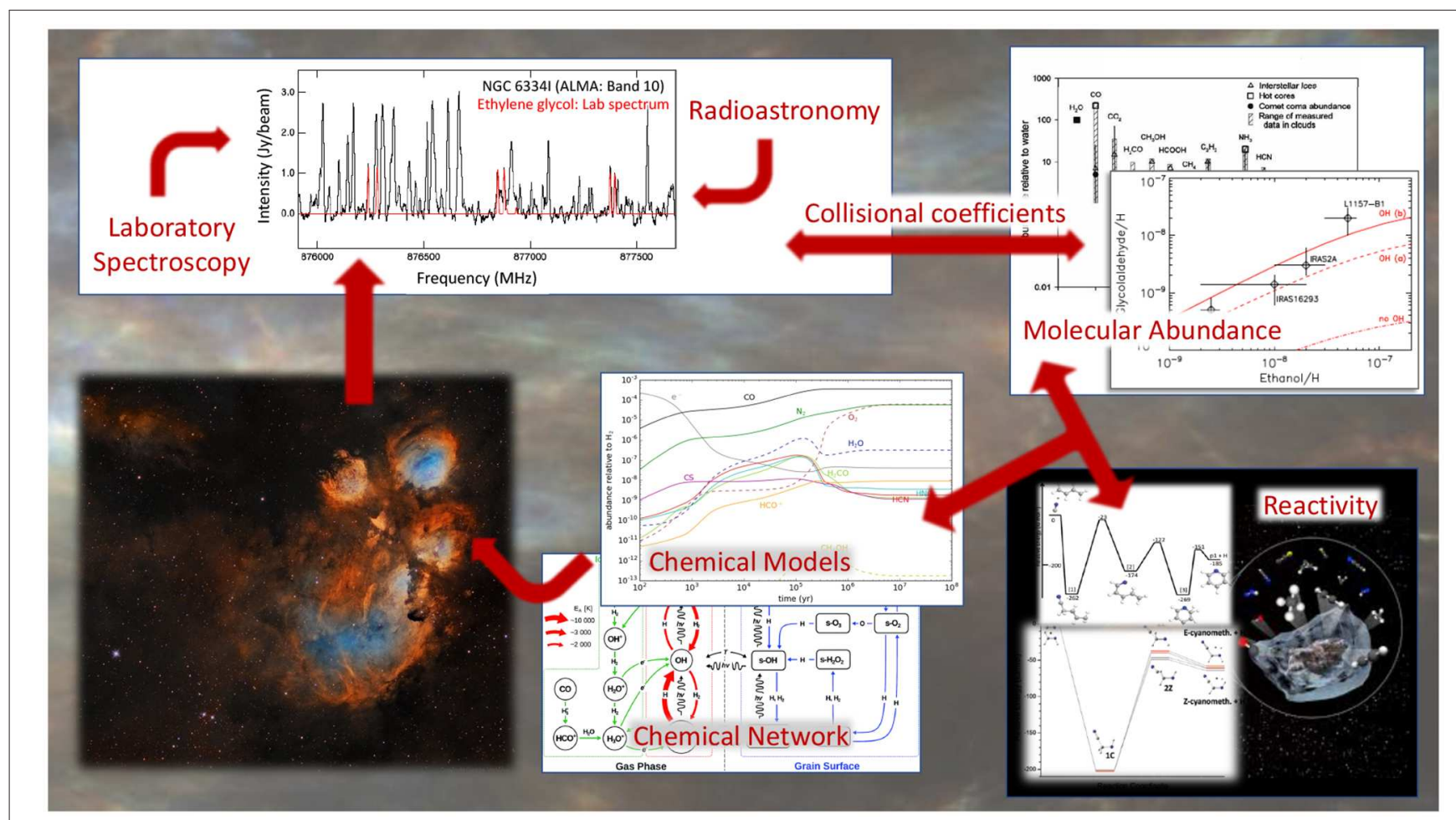

FIGURE 1 | Grand challenge 1: The stakeholders and their interplay. 
67P/Churyumov-Gerasimenko comet; Hadraoui et al., 2019), efforts to detect it in the ISM have so far failed. However, this has largely been interpreted as meaning that glycine is indeed present in the ISM, but its rotational transitions are just too weak for identification in the confusion limit of astronomical surveys. Can we find indirect ways to confirm its presence? To answer this question, a deep knowledge of chemical reactivity in space might help.

A class of compounds that deserve a special mention is that of polycyclic aromatic hydrocarbons (PAHs), a broad class of aromatic hydrocarbons made up of fused benzene rings. They have been discovered in the most disparate astronomical environments, including, e.g., interstellar dust grains, icy moons, comets and carbonaceous meteorites. Within the so-called "PAH world hypothesis," PAHs have also been assumed to be involved in the abiogenic synthesis of biological molecules. Although this hypothesis has been largely criticized, PAHs clearly play a role in the chemical evolution of the universe (d'Ischia et al., 2019). However, we are far from a complete understanding, which presents a multifaceted challenge for the astrochemical community.

\section{GRAND CHALLENGE 2: CHEMICAL REACTIVITY}

As already mentioned, understanding the chemical processes in space is one of the main aims of astrochemistry. Molecular complexity and the formation of a star proceed hand in hand. Concerning the latter, the evolution can be summarized as follows: the primordial cloud leads to a protostellar envelope, which evolves into a protoplanetary disk and then a planetary system. This star-formation process is paralleled by an increasing complexity in the chemical composition of the gas.

In the early 1970s, gas-phase ion-molecule reactions were employed to (successfully) explain the molecular abundances observed in interstellar clouds. Later on, it was recognized the importance, even in low-temperature regimes, of gas-phase neutral-neutral reactions. However, the advance of observational capabilities has led to the detection of molecules in regions where gas-phase reactions could not contribute significantly to chemical reactivity. This marked the beginning of grain-surface chemistry, i.e., the modeling of chemical reactions occurring on dust grains. However, there is still much to be understood about the formation of molecules and, often, the proposed reaction mechanisms are controversial and inconclusive (Garrod et al., 2008; Herbst and van Dishoeck, 2009; Codella et al., 2017). Both theoretical (e.g., Fernández-Ramos et al., 2006; Puzzarini and Barone, 2020) and experimental (e.g., Abeysekera et al., 2015; Parker and Kaiser, 2017) efforts, and possibly their synergic interplay (Balucani et al., 2010), are thus required.

\section{REFERENCES}

Abeysekera, C., Joalland, B., Ariyasingha, N., Zack, L. N., Sims, I. R., Field, R. W., et al. (2015). Product branching in the low temperature reaction of $\mathrm{cn}$ with
The formation routes of each individual molecular species can be thoroughly investigated. However, it is necessary to treat a large number of chemical reactions simultaneously in order to model the chemical evolution of a given astronomical environment. For setting up the complex network of elementary reactions taking place, e.g., in interstellar clouds or planetary atmospheres, a large number of physico-chemical parameters is also required. Then, kinetic models including thousands of reactions that involve hundreds of species are needed to simulate the chemical evolution over time. This has led to the growth of different astrochemical databases [such as KIDA (Wakelam et al., 2015)] to collect the kinetic parameters required for the relevant reactions. However, the data gathered in these catalogs are incomplete, and sometimes the assumptions included in the models are questionable. We are in the presence of a gigantic puzzle with a huge number of pieces that are still missing.

\section{GRAND CHALLENGE 3: THE ORIGIN OF LIFE}

Shedding light on the chemistry occurring in space, i.e., understanding how molecules are formed and evolve, might help to set the stage for understanding the emergence of life on Earth and elsewhere. This aspect of Astrochemistry has attracted a lot of interest in all scientific community, but knowledge is still at a rather primitive stage. Two theories have been suggested so far on the emergence of life on our planet (Chyba and Sagan, 1992), exogenous delivery and endogenous synthesis, and we are far from stating with confidence if one or both of them are correct. Regardless of whether they were delivered to Earth from space or synthetized from simpler molecules, prebiotic species then evolved toward biological complexity, with astrochemistry then moving toward astrobiology. Then, it becomes impossible to place a dividing line between astrochemistry and astrobiology, with astrochemical and astrobiology challenges merging together.

\section{AUTHOR CONTRIBUTIONS}

The author confirms being the sole contributor of this work and has approved it for publication.

\section{ACKNOWLEDGMENTS}

I would like to acknowledge all my group (https://site.unibo.it/rotational-computational-spectroscopy/en) for the continuous support and fruitful discussions. I also thank Prof. Vincenzo Barone (Scuola Normale Superiore) for a critical reading of the manuscript. 
Balucani, N., Leonori, F., Petrucci, R., Stazi, M., Skouteris, D., Rosi, M., et al. (2010). Formation of nitriles and imines in the atmosphere of Titan: combined crossed-beam and theoretical studies on the reaction dynamics of excited nitrogen atoms $\mathrm{N}\left({ }^{2} \mathrm{D}\right)$ with ethane. Faraday Discuss. 147, 189-216. doi: $10.1039 / \mathrm{c} 004748 \mathrm{a}$

Belloche, A., Garrod, R. T., Müller, H. S. P., and Menten, K. M. (2014). Detection of a branched alkyl molecule in the interstellar medium: iso-propyl cyanide. Science 345, 1584-1587. doi: 10.1126/science.1256678

Cheung, A. C., Rank, D. M., Townes, C. H., Thornton, D. D., and Welch, W. J. (1968). Detection of $\mathrm{nh}_{3}$ molecules in the interstellar medium by their microwave emission. Phys. Rev. Lett. 21, 1701-1705. doi: 10.1103/PhysRevLett.21.1701

Cheung, A. C., Rank, D. M., Townes, C. H., Thornton, D. D., and Welch, W. J. (1969). Detection of water in interstellar regions by its microwave radiation. Nature 221, 626-628. doi: 10.1038/221626a0

Chyba, C., and Sagan, C. (1992). Endogenous production, exogenous delivery and impact-shock synthesis of organic molecules: an inventory for the origins of life. Nature 355:125. doi: 10.1038/355125a0

Codella, C., Ceccarelli, C., Caselli, P., Balucani, N., Barone, V., Fontani, F., et al. (2017). Seeds of life in space (SOLIS)-II. Formamide in protostellar shocks: evidence for gas-phase formation. Astron. Astrophys. 605:L3. doi: 10.1051/0004-6361/201731249

d'Ischia, M., Manini, P., Moracci, M., Saladino, R., Ball, V., Thissen, H., et al. (2019). Astrochemistry and astrobiology: materials sciencein wonderland? Int. J. Mol. Sci. 20:4079. doi: 10.3390/ijms20174079

Fernández-Ramos, A., Miller, J. A., Klippenstein, S. J., and Truhlar, D. G. (2006). Modeling the kinetics of bimolecular reactions. Chem. Rev. 106, 4518-4584. doi: $10.1021 / \mathrm{cr} 050205 \mathrm{w}$

Garrod, R. T., Weaver, S. L. W., and Herbst, E. (2008). Complex chemistry in starforming regions: an expanded gas-grain warm-up chemical model. Astrophys. J. 682, 283-302. doi: 10.1086/588035

Goldsmith, P. F., and Langer, W. D. (1999). Population diagram analysis of molecular line emission. Astrophys. J. 517, 209-225. doi: 10.1086/307195

Hadraoui, K., Cottin, H., Ivanovski, S. L., Zapf, P., Altwegg, K., Benilan, Y., et al. (2019). Distributed glycine in comet 67p/churyumov-gerasimenko. Astron. Astrophys. 630:A32. doi: 10.1051/0004-6361/201935018

Hartmann, J. (1904). Investigations on the spectrum and orbit of delta Orionis. Astrophys. J. 19, 268-286. doi: 10.1086/141112

Herbst, E., and van Dishoeck, E. F. (2009). Complex organic interstellar molecules. Ann. Rev. Astron. Astrophys. 47, 427-480. doi: 10.1146/annurev-astro-082708-101654

Holdship, J., Viti, S., Jiménez-Serra, I., Makrymallis, A., and Priestley, F. (2017). UCLCHEM: a gas-grain chemical code for clouds, cores, and c-shocks. Astron. J. 154:38. doi: 10.3847/1538-3881/aa773f

Kuan, Y.-J., Charnley, S. B., Huang, H.-C., Tseng, W.-L., and Kisiel, Z. (2003). Interstellar glycine. Astrophys. J. 593, 848-867. doi: 10.1086/375637

Kvenvolden, K. A., Lawless, J., Pering, K., Peterson, E., Flores, J., Ponnamperuma, C., et al. (1970). Evidence for extraterrestrial amino-acids and hydrocarbons in the murchison meteorite. Nature 228:9230926. doi: 10.1038/228923a0

McGuire, B. A. (2018). 2018 census of interstellar, circumstellar, extragalactic, protoplanetary disk, and exoplanetary molecules. Astrophys. J. Suppl. Ser. 239:17. doi: 10.3847/1538-4365/aae5d2
McGuire, B. A., Carroll, P. B., Loomis, R. A., Finneran, I. A., Jewell, P. R., Remijan, A. J., et al. (2016). Discovery of the interstellar chiral molecule propylene oxide $\left(\mathrm{CH}_{3} \mathrm{CHCH}_{2} \mathrm{O}\right)$. Science 352, 1449-1452. doi: 10.1126/science.aae0328

McKellar, A. (1940). Evidence for the molecular origin of some hitherto unidentified interstellar lines. Publ. Astron. Soc. Pac. 52:187. doi: $10.1086 / 125159$

Melosso, M., Melli, A., Puzzarini, C., Codella, C., Spada, L., Dore, L., et al. (2018). Laboratory measurements and astronomical search for cyanomethanimine. Astron. Astrophys. 609:A121. doi: 10.1051/0004-6361/201731972

Müller, H. S. P., Schlöder, F., Stutzki, J., and Winnewisser, G. (2005). The cologne database for molecular spectroscopy, cdms: a useful tool for astronomers and spectroscopists. J. Mol. Struct. 742, 215-227. doi: 10.1016/j.molstruc.2005.01.027

Nash, A. G. (1990). The abundance ratio of formaldehyde to ammonia in molecular clouds observed toward radio continuum sources. Astrophys. J. Suppl. Ser. 72:303. doi: $10.1086 / 191418$

Parker, D. S. N., and Kaiser, R. I. (2017). On the formation of nitrogen-substituted polycyclic aromatic hydrocarbons (NPAHs) in circumstellar and interstellar environments. Chem. Soc. Rev. 46, 452-463. doi: 10.1039/C6CS00714G

Puzzarini, C., and Barone, V. (2020). The challenging playground of astrochemistry: an integrated rotational spectroscopy-quantum chemistry strategy. Phys. Chem. Chem. Phys. 22, 6507-6523. doi: 10.1039/D0CP00561D

Snyder, L. E., and Buhl, D. (1971). Observations of radio emission from interstellar hydrogen cyanide. Astrophys. J. Lett. 163:L47. doi: 10.1086/180664

Snyder, L. E., Buhl, D., Zuckerman, B., and Palmer, P. (1969). Microwave detection of interstellar formaldehyde. Phys. Rev. Lett. 22, 679-681. doi: 10.1103/PhysRevLett.22.679

Tennyson, J. (Ed.). (2005). Astronomical Spectroscopy. London: Imperial College Press. doi: 10.1142/p371

Trumpler, R. J. (1930). Absorption of light in the galactic system. Publ. Astronom. Soc. Pac. 42:214. doi: 10.1086/124039

van Dishoeck, E. F. (2017). Astrochemistry: overview and challenges. Proc. Int. Astron. Union 13, 3-22. doi: 10.1017/S1743921317011528

Wakelam, V., Loison, J.-C., Herbst, E., Pavone, B., Bergeat, A., Béroff, K., et al. (2015). The 2014 KIDA network for interstellar chemistry. Astrophys. J. Suppl. Ser. 217:20. doi: 10.1088/0067-0049/217/2/20

Wilson, R. W., Jefferts, K. B., and Penzias, A. A. (1970). Carbon monoxide in the Orion Nebula. Astrophys. J. Lett. 161:L43. doi: 10.1086/180567

Yamamoto, S. (Ed.). (2017). Introduction to Astrochemistry (Chemical Evolution from Interstellar Clouds to Star and Planet Formation). Springer. Tokyo. doi: 10.1007/978-4-431-54171-4_9

Conflict of Interest: The author declares that the research was conducted in the absence of any commercial or financial relationships that could be construed as a potential conflict of interest.

Copyright (c) 2020 Puzzarini. This is an open-access article distributed under the terms of the Creative Commons Attribution License (CC BY). The use, distribution or reproduction in other forums is permitted, provided the original author(s) and the copyright owner(s) are credited and that the original publication in this journal is cited, in accordance with accepted academic practice. No use, distribution or reproduction is permitted which does not comply with these terms. 